OPEN ACCESS

Edited by:

Christian Gonzalez-Billault,

University of Chile, Chile

Reviewed by:

Constanze I. Seidenbecher, Leibniz Institute for Neurobiology (LG),

Germany

Vladimir Sytnyk

University of New South Wales,

Australia

*Correspondence:

Camila Scorticati

cscorticati@iib.unsam.edu.ar

tThese authors have contributed equally to this work and share first authorship

Received: 31 January 2021 Accepted: 07 April 2021 Published: 04 May 2021

Citation:

León A, Aparicio Gl and Scorticati $C$ (2021) Neuronal Glycoprotein M6a: An Emerging Molecule in Chemical Synapse

Formation and Dysfunction.

Front. Synaptic Neurosci. 13:661681. doi: 10.3389/fnsyn.2021.661681

\section{Neuronal Glycoprotein M6a: An Emerging Molecule in Chemical Synapse Formation and Dysfunction}

\author{
Antonella Leónt, Gabriela I. Aparicioł ${ }^{+}$and Camila Scorticati* \\ Instituto de Investigaciones Biotecnológicas "Rodolfo A. Ugalde", Universidad Nacional de San Martín and Consejo Nacional \\ de Investigaciones Científicas y Técnicas (IIBio-UNSAM-CONICET), Buenos Aires, Argentina
}

The cellular and molecular mechanisms underlying neuropsychiatric and neurodevelopmental disorders show that most of them can be categorized as synaptopathies-or damage of synaptic function and plasticity. Synaptic formation and maintenance are orchestrated by protein complexes that are in turn regulated in space and time during neuronal development allowing synaptic plasticity. However, the exact mechanisms by which these processes are managed remain unknown. Large-scale genomic and proteomic projects led to the discovery of new molecules and their associated variants as disease risk factors. Neuronal glycoprotein M6a, encoded by the GPM6A gene is emerging as one of these molecules. M6a has been involved in neuron development and synapse formation and plasticity, and was also recently proposed as a gene-target in various neuropsychiatric disorders where it could also be used as a biomarker. In this review, we provide an overview of the structure and molecular mechanisms by which glycoprotein M6a participates in synapse formation and maintenance. We also review evidence collected from patients carrying mutations in the GPM6A gene; animal models, and in vitro studies that together emphasize the relevance of $\mathrm{M6a}$, particularly in synapses and in neurological conditions.

Keywords: GPM6A, neuronal plasticity, synaptopathy, synaptic interactome, PLP family

\section{INTRODUCTION}

The excitatory synapses are specific neuron-neuron communications between axon and dendritic processes that orchestrate the information stream and storage in the brain (Tu et al., 2018). Synapse formation involves a complex series of events with at least three primary stages: axon elongation and guidance by which axons reach their target area; synaptic specificity governed by an appropriate association of synaptic molecules, and synaptogenesis which creates functional synapses (Shen and Cowan, 2010).

Functional synapses are supported by specialized protein complexes, whose function is regulated in time and space during neuronal development, allowing effective synaptic plasticity (Torres et al., 2017). The cellular and molecular mechanisms underlying neuropsychiatric and neurodegenerative disorders reveal that most of them can be classified as synaptopathies (Sengpiel, 2018), however, their full understanding so far remains unknown. Large scale genome-wide association studies (GWAS, reviewed in Claussnitzer et al., 2020) promote the study of additional candidates. Candidates arise either as genetic vulnerability or susceptibility loci allowing researchers to explore 
how they might be involved in the molecular mechanisms governing neurological diseases with complex etiology and heterogeneous genetic predisposition.

Neuronal glycoprotein M6a, encoded by the GPM6A gene, is a member of the tetraspan proteolipid protein (PLP) family together with PLP/DM20 and M6b. Since its discovery in 1992 (Baumrind et al., 1992; Lagenaur et al., 1992), M6a has emerged as one of many proteins involved in neuron development, synapse plasticity, and as a key component in various neuropsychiatric disorders (Michibata et al., 2009; El-Kordi et al., 2013; Gregor et al., 2014; Fuchsova et al., 2015). This review provides a quick overview of the structure and molecular mechanisms by which M6a participates in synapse formation and maintenance. Moreover, we review evidence collected from patients carrying mutations within GPM6A; animal models, and in vitro studies that highlight the relevance of M6a, particularly in synapses and related neurological conditions.

\section{GENE, PROTEIN, AND STRUCTURAL DOMAINS}

The PLP family members are integral membrane proteins with a conserved topology: four transmembrane domains (TMDs), two extracellular loops (EC1 and EC2), one intracellular loop (IC), and the $\mathrm{N}$ - and C-termini both at the cytoplasmic face (Figure 1A). M6a exhibits low sequence identity with both PLP (38\%) and M6b (52\%), however, the TMDs are highly conserved (Greer and Lees, 2002; Fernandez et al., 2010).

Human glycoprotein M6a is encoded by a 369,731 kb gene organized into seven exons and located at chromosome 4q34.2. The full-length gene encodes a 278 amino acid membrane protein with a molecular mass of approximately $32 \mathrm{kDa}$ (Olinsky et al., 1996). The amino acid sequence of M6a is highly conserved within mammals (more than $98 \%$ of identity). Post-translational modifications in $\mathrm{M6a}$ are summarized in Figure 1A. M6a has seven potential phosphorylation sites, and some of them are responsible for specific features described below. M6a has four cysteine residues within its EC2, critical for its folding and function, particularly C174 and C192 are linked by a disulfide bond, forming an intradomain important for protein-protein interactions (Fuchsova et al., 2009). The EC2 also contains two predicted N-glycosylation sites. Only glycosylation at N164 was experimentally corroborated, although there are no reported functions to date (Fang et al., 2016). Seven other cysteine residues close to the TMDs in the cytoplasmic side are potential palmitoylation sites, three of which (C17/18/21) - conserved within the PLP family-are necessary for M6a inclusion in lipid rafts (Honda et al., 2017; Ito et al., 2018).

GPM6A's RNA-expression rapidly increases during development in human and murine brains. M6a is a brainspecific gene with a very high level of expression, representing one of the most abundant palmitoylated proteins in the CNS (Huminiecki et al., 2003; Kang et al., 2008). By contrast, low expression was detected in the lung, spleen, ovary, and the thyroid gland (Fagerberg et al., 2014; Yu et al., 2014;
Yue et al., 2014). M6a protein levels are enriched in the hippocampus, cerebellum, striatum, and prefrontal cortex among other brain areas ${ }^{1}$. Regarding specific cell expression at the CNS, M6a is mostly placed at the cell surface of neurons and epithelial cells of the choroid plexus, but not in glial cells. The neuronal expression of M6a gives it a distinctive feature within the PLP family members as PLP is expressed only in glial cells and M6b is expressed in both neurons and glia (Werner et al., 2013).

\section{M6a AND SYNAPSE FORMATION}

\section{M6a's Role in the Presynaptic Formation}

The coordination of neuronal differentiation, axonal growth, and guidance involves timely expression of cell surface proteins and extracellular adhesion molecules escorted by structural changes in the cellular cytoskeleton (Caceres et al., 2012). During neurite outgrowth, plasma membrane proteins are directed toward neurites first, and then they are concentrated in growth cones (GCs). There, proteins will be available to respond to orientation signals and to signal the path to a specific destination (Fuentes and Arregui, 2009; Ulloa et al., 2018). Indeed, M6a was identified as an "edge-membrane antigen" (EMA) because it was found concentrated at the edge of neuronal GCs and their lamellipodia in cultured neurons from the cerebellum, cortex, and hippocampus (Baumrind et al., 1992; Lagenaur et al., 1992). Later on, M6a was found to be critical for neurite growth in a wide variety of in vitro models, from brain tissue explants to neuronal cell lines and from human to Xenopus (Lagenaur et al., 1992; Mukobata et al., 2002; Alfonso et al., 2005; Zhao et al., 2008; Michibata et al., 2009; Formoso et al., 2015a). Noteworthy, Sato et al. (2011a) observed a reduction of the axon projections in the olfactory bulb from embryonic brains at E14.5 of Gpm6a knockout mice. Besides, double knockout mice for Gpm6a and Gpm6b show decreased axon elongation and a thinner corpus callosum which could be rescued by forced expression of M6 proteins (Mita et al., 2015).

To date, redundant signaling pathways of Src/MAPK/ERK, $\mathrm{PKC}$, and PI3K/AKT kinases mediate M6a-induced neurite extension in hippocampal neurons and neuroblastoma cells (Mukobata et al., 2002; Scorticati et al., 2011; Formoso et al., 2015a). The phosphorylation of tyrosine 251 close to M6a's C-terminus is critical for neuritogenesis (Formoso et al., 2015a). By contrast, cells expressing a truncated form of M6a lacking the N-terminus did not show neurite outgrowth inhibition (Mita et al., 2015). The direct association of M6a's C-terminus with coronin1Aa cytoskeleton adaptor molecule-verify its essential role in the extension of projections (Alvarez Julia et al., 2016; Martorella et al., 2017). Furthermore, Honda et al. (2017) identified that M6a induces neuron polarity through the Rufy3-Rap2-STEF/Tiam2 pathway.

\footnotetext{
${ }^{1}$ www.proteinatlas.org
} 

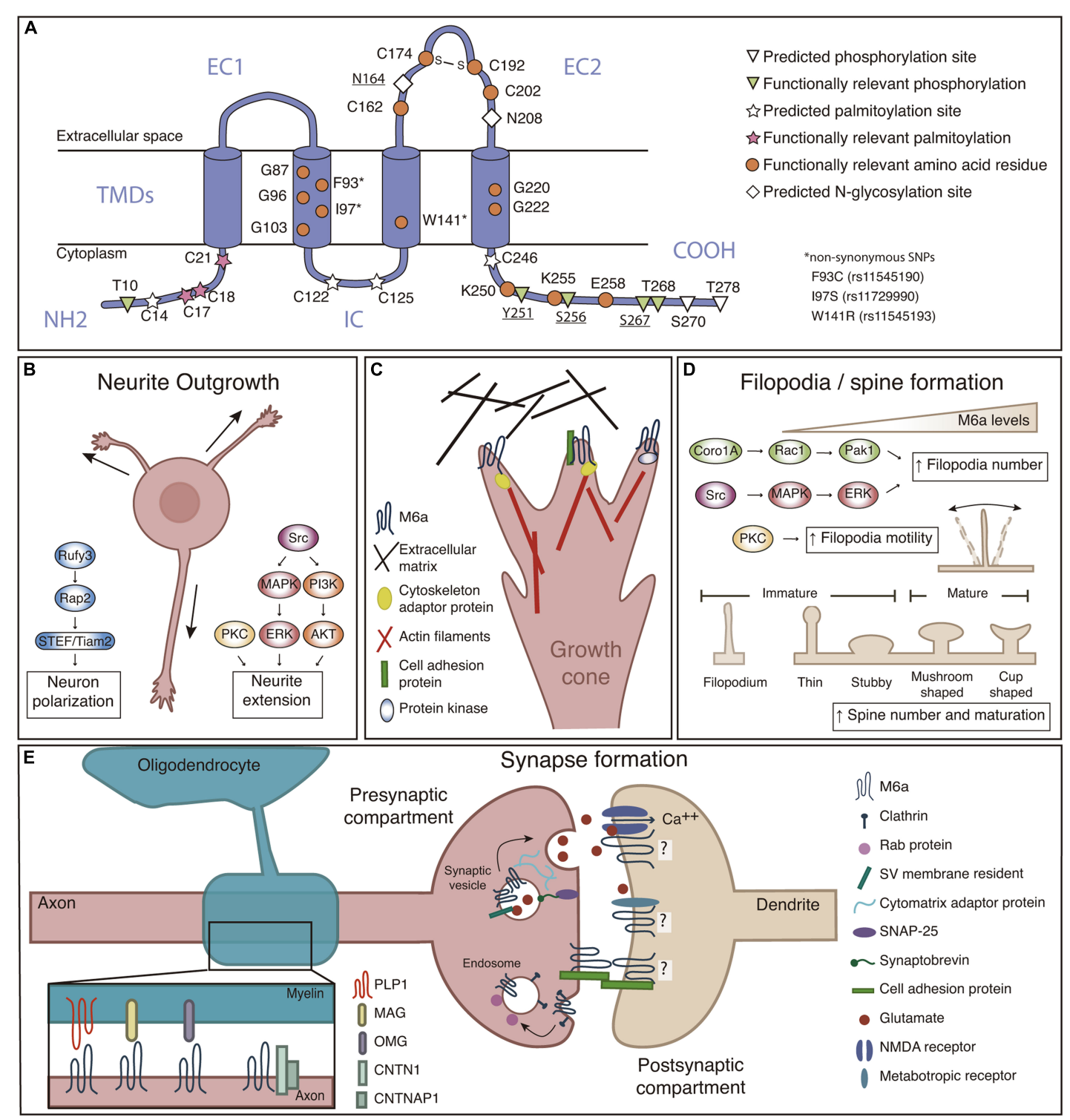

FIGURE 1 | (A) M6a structural features. M6a's topological computational model predicts four transmembrane domains (TMDs), two extracellular loops (a small one-EC1- and a large one-EC2-), an intracellular loop (IC), and N- and C-terminal ends facing the cell cytoplasm (NH2 and COOH). Predicted sites for palmitoylation (C14, C17, C18, C21, C122, C125, and C246), N-glycosylation (N164 and N208), and phosphorylation (T10, Y251, S256, S267, T268, S270, and T278) are highlighted; bold underlined sites indicate post-translational modifications which are experimentally validated. Orange circles represent amino acid residues relevant for M6a function. (B) M6a promotes neurite outgrowth and neuronal polarization, which involves the activation of SrC/MAPK/ERK, PKC and PI3K/AKT, and Rufy3-Rap2-STEF/Tiam2 signaling pathways, respectively. (C) We hypothesize that M6a function depends on its association with partner proteins in specific membrane microdomains. These associations, induce auto/phosphorylation in specific C-terminal residues facing the cytoplasm, and finally promote neuronal plasticity (Fuchsova et al., 2009; Scorticati et al., 2011; Formoso et al., 2015a,b; Garcia et al., 2017; Honda et al., 2017; Aparicio et al., 2020). For example, M6a can associate with extracellular matrix proteins such as brevican and tenascin C through its extracellular loops in trans and/or with cell adhesion proteins (NCAM and NPTN) through its extracellular domains in cis, triggering its phosphorylation and therefore the recruitment of adapter proteins (such as Rap) and the activation of protein kinases that finally promote the reorganization of the cytoskeleton. (D) M6a promotes filopodia formation and motility through a mechanism that involves the activation of the Coro1A/Rac1/Pak1 and Src/MAPK/ERK, and PKC pathways, respectively. M6a also participates in spine formation and maturation of dendritic protrusions. (E) Left: Recently proposed interactions between M6a and myelin sheath proteins (PLP1, MAG, and OMG), and myelinated-axon proteins CNTN1 and CNTNAP1. Right: M6a is located at the presynaptic compartment plasma membrane, synaptic vesicle-SV-and endosomal membranes. M6a also interacts with postsynaptic glutamate receptors (like NMDA-R1 and GRM1) but its localization to the postsynaptic compartment was not experimentally confirmed yet. M6a, glycoprotein M6a; Src, Src kinase; MAPK, mitogen-activated protein kinase; ERK, extracellular signal-regulated kinase; PKC, protein kinase C; PI3K, phosphatidylinositide 3-kinase; AKT, serine/threonine-protein kinase; Rap2, Ras-related protein-2; Rufy3, Rufy 3 protein; STEF/Tiam2, T-lymphoma invasion and metastasis-inducing protein 2; NCAM, Neural cell adhesion molecule; NPTN, neuroplastin; Coro1A, coronin-1A; Rac1, Ras-related C3 botulinum toxin substrate 1; Pak1, p21-activated kinase 1; PLP1, proteolipid protein 1; MAG, myelin-associated glycoprotein; OMG, oligodendrocyte-myelin glycoprotein; CNTN1, contactin-1; CNTNAP1, contactin-associated protein 1; NMDA-R1, N-methyl-D-aspartate receptor 1; GRM1, metabotropic glutamate receptor 1; SNAP25, Synaptosomal-Associated Protein, $25 \mathrm{kDa}$. 
Recent proteomic studies identified proteins associated with M6a that participate in neurite outgrowth and axon elongation (Figures 1B,C; Sato et al., 2011a; Huttlin et al., 2017; Aparicio et al., 2020; Haenig et al., 2020; Pourhaghighi et al., 2020). To emphasize some examples, we divided them into 3 groups: (i) Cytoplasmic proteins: which include cytoskeleton adaptor molecules and cytoskeleton organization pathways which might interact with M6a's cytoplasmic tails as Ras kinases 2, DiRas2 (Grunewald et al., 2021) and the kinases mentioned above. (ii) Cell adhesion molecules (CAMs): which mediate recognition of cellular targets, bind the cell surface to the extracellular matrix (ECM), and maintain cell shape. Examples of these molecules that could interact with M6a's extracellular loops or its transmembrane domains are neuronal cell adhesion molecule (NCAM) (Neugebauer et al., 1988), and neuroplastin, NPTN (Beesley et al., 2014). (iii) ECM proteins: which only could interact with M6a's extracellular loops: brevican (BCAN) (Miura et al., 2001), and tenascin C (TNC) (Andrews et al., 2009).

Presynaptic boutons are terminal specializations of the axon, which contain synaptic vesicles (SVs) filled with neurotransmitters. This specific compartmentalization involves the coordination of both SVs and presynaptic active zone proteins, which define regions in the membrane for the release of neurotransmitters to the synaptic cleft (Yue et al., 2014). Thus, there are three main types of proteins (i) residents of SV's membrane, such as synaptophysin (Egbujo et al., 2016), (ii) filament or adaptor proteins of the cytomatrix, such as piccolo and bassoon (Hida and Ohtsuka, 2010), and (iii) those that participate in synaptic vesicle exocytosis such as synaptosomal associated protein 25 (SNAP25) (Antonucci et al., 2016; Figure 1E). Roussel et al. (1998) revealed that M6a was distributed at the presynaptic membrane, in particular, on the membrane of SVs which was confirmed by proteomic analysis (Takamori et al., 2006; Taoufiq et al., 2020). Besides, M6a was also found at glutamatergic nerve terminals in the cerebellum and cerebral cortex but not in GABAergic neurons of adult brain mice. This specific-excitatory preference was also confirmed in the hippocampal formation in which M6a colocalizes with the vesicular glutamate transporter VGLUT (Cooper et al., 2008).

Recently, M6a has also been associated with other SVs and presynaptic active zone-PAZ - residents proteins, like synaptic vesicle protein $2 \mathrm{~B}$ (SV2B), piccolo, bassoon, and synapsin 1 (Aparicio et al., 2020). Overexpression of M6a in hippocampal neurons showed a significant increment of synaptophysin puncta correlating with an increase in the number of synapses. On the contrary, neurons subjected to siRNA depletion of M6a and neurons overexpressing a truncated form of M6a at the EC2 loop exhibited a decreased number of synaptophysin puncta (Alfonso et al., 2005; Fuchsova et al., 2009; Formoso et al., 2016). Moreover, M6a internalizes and recycles back to the cell membrane via clathrin-mediated endocytosis, and localizes in Rab5, Rab7, and Rab11 positive endosomes, just like the case of SVs recycling pathways (Wu et al., 2007; Formoso et al., 2016; Garcia et al., 2017; Rosas et al., 2018). Indeed, inducing M6a acute internalization correlated with a decrease of both the number of synaptophysin puncta and synapses
(Garcia et al., 2017), suggesting that M6a might be playing an active role in SV/neurotransmitter release.

\section{M6a's Role in the Postsynaptic Formation}

Straight opposed to the presynaptic terminal is the postsynaptic target. Dendritic spines, the main postsynaptic compartment, are protrusions from the dendrite shaft that receive the information from axonal terminals through different neurotransmitter receptors. Dendritic spines can show dynamic changes in number, size, shape, and movement which allow synaptic rearrangements to take place (Tonnesen and Nagerl, 2016). This plastic feature is a critical substrate for functional plasticity during pruning or learning and memory, and also for synaptic dysfunction as observed in neurodegenerative and neuropsychiatric conditions including Alzheimer's Disease (AD), autism spectral disorders (ASD), schizophrenia, and depression (Penzes et al., 2011; Bian et al., 2015; Ozcan, 2017).

According to its size and shape, dendritic spines can be classified as thin, stubby, mushroom, and cup-shaped. Moreover, according to their functionality, dendritic protussions can also be classified as immature (filopodia, thin and stubby) or mature (mushroom and cup-shaped) (Figure 1D). Three possible models have been proposed to explain how a synapse is formed: (i) Sotelo's model describes that a synapse could arise when an immature spine is contacted by the axon terminal inducing its development toward the mushroom type (Sotelo et al., 1975), (ii) Miller/Peters's model proposes that a presynaptic terminal directly contacts with the dendrite shaft, inducing spine outgrowth (Miller and Peters, 1981), and (iii) Filopodial model: where dendritic filopodia may actively initiate synaptogenic contacts by contacting a presynaptic terminal, thereby inducing its stabilization and subsequently maturation to the mushroom type (Yuste and Bonhoeffer, 2004; Ziv and Fisher-Lavie, 2014).

M6a has been widely described to be involved in filopodia/spine formation in different cell culture models (Alfonso et al., 2005; Sato et al., 2011b; Scorticati et al., 2011; Formoso et al., 2015b; Alvarez Julia et al., 2016; Formoso et al., 2016; Rosas et al., 2018). Two pathways have been implicated by which M6a increases filopodia density. M6a overexpression induces the activation by phosphorylation of the intracellular cascade involving the Src and MAPK/ERK pathway; and the localization of M6a within lipid rafts is compatible with this (Scorticati et al., 2011). Likewise, signaling pathways that include Rac1 and Pak1 activation through coronin1A facilitate M6ainduced filopodium formation (Alvarez Julia et al., 2016). There are key domains required for M6a to induce filopodia/spine formation (Figure 1D): (i) TMDs homotypic-interactions, especially commanded by particular glycine residues at TMD2 and TMD4 (Formoso et al., 2015b, 2016), (ii) a disulfide bridge at EC2 loop formed between cysteine residues C174 and C192 (Fuchsova et al., 2009), and (iii) C-terminal domain residues K250/K255/E258 (Rosas et al., 2018). Although the N-terminus is not involved in filopodia induction (Mita et al., 2015; Rosas et al., 2018), phosphorylation of certain serine/threonine residues at 
$\mathrm{N}$-and C-termini, T10/S256/S267/T268, proved to be necessary for filopodial motility (Brocco et al., 2010).

Although M6a induces dendritic protrusions plasticity, whether or not it is placed at the postsynaptic membrane is undetermined. For instance, M6a was identified at presynaptic membranes and enriched in glutamatergic synaptic vesicles docked to the presynaptic active zone (Roussel et al., 1998; Boyken et al., 2013). Conversely, M6a was detected in a proteomic analysis of enriched postsynaptic membrane fractions from mice brains, suggesting a postsynaptic localization for M6a (Reim et al., 2017). Besides, we described that M6a co-immunoprecipitated with integral components of the postsynaptic membranes such as metabotropic glutamate receptor 1 (GRM1), voltage-dependent anion channel 1 (VDAC1), and N-methyl-D-aspartate receptor type 1, NMDA-R1 (GR1A1) (Aparicio et al., 2020). Indeed, M6aoverexpressing neurons exhibited an increase in the number of NMDA-R1 clusters, whilst truncated forms of M6a or its depletion decreased the number of NMDA-R1 clusters (Formoso et al., 2016; Garcia et al., 2017). The colocalization of M6a and NMDA-R1 suggests that M6a acts as a scaffold protein assembling proteins and lipids to form a signaling platform on the neuronal surface (Wu et al., 2007; Scorticati et al., 2011). However, to determine whether M6a is indeed located at the postsynaptic membrane techniques such as cryo-electron microscopy and super-resolution microscopy are needed to avoid contaminations of biochemical-based techniques (Liu et al., 2019; Nosov et al., 2020).

\section{M6a'S POTENTIAL ROLE IN NEURON-GLIA INTERACTION}

M6a is enriched within cerebellar parallel and hippocampal mossy fibers, whose axons remain unmyelinated also in adulthood (Cooper et al., 2008). Nonetheless, new data suggest that M6a could participate in neuron-glia interactions. This subject exceeds the topics covered in this review, and no functional experiments were reported yet, but we would like to highlight a few insights. Pourhaghighi et al. (2020) identified associated protein complexes in adult mammal brains and built BraInMap'2. M6a was found within a complex of 30 interacting proteins, some of which are myelin sheath proteins, including the main myelin glycoproteins PLP and MAG; or are myelin sheath associated proteins like contactin 1 and contactin associated protein 1. In agreement, we identified 20 myelin proteins in the co-immunoprecipitation complexes formed by M6a's extracellular domains and rat hippocampal samples, in which PLP was experimentally confirmed (Aparicio et al., 2020). Also, Jahn et al. (2020) identified M6a in the myelin sheath of post mortem human brains.

\section{M6a IN SYNAPSE FUNCTION AND DYSFUNCTION}

The evidence reviewed so far highlights an important role of M6a in neuronal development and synaptic plasticity. However,

\footnotetext{
${ }^{2}$ www.bu.edu/dbin/cnsb/mousebrain
}

few reports interrogate about the role of M6a on active synapses. All of them are based on determining the cluster number of synaptophysin and/or the number of clusters between synaptophysin and NMDA-R1 on in vitro models (Alfonso et al., 2005; Fuchsova et al., 2009; Formoso et al., 2016; Garcia et al., 2017). For instance, endogenous M6a acute depletion using siRNA or treatments with M6a-mAb neutralizing antibodies dramatically decrease the number of synaptophysin clusters, or synaptophysin and NMDA-R1 colocalization clusters (Alfonso et al., 2005; Garcia et al., 2017). The latter suggests that M6a is not only required for the formation of synapses but also their maintenance. Evidence from M6a's relevance in vivo comes from the observation that several GPM6A variants and inadequate expression levels were associated with several neuropsychiatric disorders (see Table 1), thus highlighting that the impairment of M6a function contributes to disease onset or increases the risk of susceptibility.

Chronic stress induces behavioral changes in mammals, which can be evidenced by mood disorders including anxiety, claustrophobia, and depression. In this context mRNA levels of Gpm6a were decreased in the hippocampus of chronically stressed animals (Alfonso et al., 2002, 2004a,b); and then rescued with the administration of antidepressants (Alfonso et al., 2006). These are consistent with findings where chronically stress animals presented a reduction of the dendritic arborization and structural changes in the mossy fiber terminals (Cooper et al., 2008). In humans, GPM6A mRNA levels were also decreased in the hippocampus of depressed patients who committed suicide (Fuchsova et al., 2015). Furthermore, depressed patients treated with serotonin reuptake inhibitors showed a reduction of M6a levels in saliva compared to depressed patients under benzodiazepine treatment or no treatment at all. As a result, M6a was proposed as a mood disorder biomarker (Monteleone et al., 2020).

Gpm6a knockout mice lack obvious behavioral abnormalities but when subjected to moderate restrain stress, they presented a claustrophobia-like phenotype. These findings encouraged El-Kordi and collaborators to investigate GPM6A variants within claustrophobic patients. In their study they found a $3^{\prime}$-untranslated region variant that produced a functional mRNA that could not be silenced by neuronal miR124, thereby losing the physiological regulation of the gene (El-Kordi et al., 2013). Gregor et al. (2014) identified a de novo duplication of the GPM6A gene in a patient with learning disabilities and behavioral anomalies. Furthermore, Rao-Ruiz et al. (2015) revealed that in mice subjected to a contextual fear-memory learning task M6a was differentially up-regulated $4 \mathrm{~h}$ later. Until now, there was no direct relationship between M6a levels and the molecular processes underlying memory and learning formation. However, these independent findings suggest that this may warrant further investigation.

The intrinsic genetic program responsible for synapse formation and maintenance involves a correct pruning of the dendritic spines. An abnormal dendritic spine pruning during development and in adulthood contributes to the generation of synaptopathies such as ASD, schizophrenia, 
TABLE 1 | Summary of GPM6A gene variants and expression levels related to disorders.

\begin{tabular}{|c|c|c|c|c|c|c|}
\hline Alteration & Species & Approach & Sample & Study & Result & References \\
\hline \multirow[t]{4}{*}{ Chronic Stress } & Rats & Restraint stress & $\mathrm{HP}$ & mRNA & $\downarrow$ & Alfonso et al., 2002 \\
\hline & Shrews & Psychosocial stress & $\mathrm{HP}$ & mRNA & $\downarrow$ & Alfonso et al., 2004a,b \\
\hline & Mice & Restraint stress & $\mathrm{HP}$ & mRNA & $\downarrow$ & Alfonso et al., 2006 \\
\hline & Mice & Prenatal stress & $\mathrm{HP}$ & mRNA & $\uparrow$ & Monteleone et al., 2014 \\
\hline \multirow[t]{2}{*}{ Claustrophobia } & Mice & Gpm6a-/- & & Behavioral test & $\downarrow$ & El-Kordi et al., 2013 \\
\hline & Humans & & & Genotyping & $\begin{array}{l}\text { GPM6A variant } \\
\text { 3`UTR }\end{array}$ & \\
\hline Neuroticism & Humans & & & GWAS & rs17611770 & Nagel et al., 2018 \\
\hline \multirow[t]{2}{*}{ Depression } & Humans & & $\mathrm{HP} / \mathrm{PFC}$ & mRNA & $\downarrow$ & Fuchsova et al., 2015 \\
\hline & Humans & & Saliva & ELISA & $\mathrm{ND}$ and $\uparrow$ & Monteleone et al., 2020 \\
\hline Bipolar Disorder & Humans & & & GWAS & rs17599018 & Greenwood et al., 2012 \\
\hline \multirow[t]{2}{*}{ Schizophrenia } & Humans & with depression & & Genotyping & rs10520303 & Boks et al., 2008 \\
\hline & Humans & & & GWAS & $\begin{array}{l}\text { rs1106568, } \\
\text { rs62334820, } \\
\text { rs13142920, } \\
\text { rs7673823, } \\
\text { rs6846161 }\end{array}$ & $\begin{array}{l}\text { Schizophrenia Working } \\
\text { Group of the Psychiatric } \\
\text { Genomics Consortium, } \\
\text { 2014; Li et al., 2017; } \\
\text { Pardiñas et al., 2018; } \\
\text { Lam et al., } 2019\end{array}$ \\
\hline Learning disability & Humans & & & $\begin{array}{l}\text { Genotyping } \\
\text { Microarray }\end{array}$ & GPM6A duplication & Gregor et al., 2014 \\
\hline \multirow[t]{4}{*}{ Alzheimer Disease } & Mice & APP/PS1 mice & $\mathrm{OB}$ & Proteomics & $\uparrow$ & Lachén-Montes et al., 2016 \\
\hline & Humans & & $\mathrm{HP}$ & mRNA microarray & $\downarrow$ & Xu et al., 2006 \\
\hline & Humans & EVs & Cortical gray matter & Proteomics & $\uparrow$ & $\begin{array}{l}\text { Muraoka et al., 2020; } \\
\text { Quiroz-Baez et al., } 2020\end{array}$ \\
\hline & Humans & EVs & CSF & Proteomics & $\uparrow$ & Chiasserini et al., 2020 \\
\hline \multirow[t]{2}{*}{$\begin{array}{l}\text { Autistic spectrum } \\
\text { disorder }\end{array}$} & Mice & $\begin{array}{l}\text { PSD membrane } \\
\text { fractions }\end{array}$ & Striatum and HP & Proteomics & ND & Reim et al., 2017 \\
\hline & Humans & & $\mathrm{CB}$ & Proteomics & $\uparrow$ & Abraham et al., 2019 \\
\hline
\end{tabular}

or AD (Penzes et al., 2011). For instance, subjects with ASD have deficits in social interactions, disruption of oral communication, and present repetitive behavior; correlated with an elevated number of dendritic spines and a late onset of the pruning process (Toro et al., 2010). Interestingly, a proteomic analysis done by Abraham et al. (2019) revealed that M6a levels were increased in samples from the cerebellum of patients with ASD. Moreover, proteomic studies showing altered M6a levels in both animal models and patients with $\mathrm{AD}$ contribute to a possible role in memory decline (Xu et al., 2006; Lachén-Montes et al., 2016; Chiasserini et al., 2020; Muraoka et al., 2020). Also, M6a is downregulated in the hippocampal formation whereas it is enriched in extracellular vesicles from cerebrospinal fluid (CSF) samples of $\mathrm{AD}$ patients suggesting an active secretion of M6a during $\mathrm{AD}$ progression (Xu et al., 2006; Chiasserini et al., 2020). Indeed, Muraoka et al. (2020) proposed M6a, together with ANX5, VGF, and ACTZ as a biomarker for monitoring AD progression from CSF samples (Muraoka et al., 2020).

One of the goals of genome-wide association studies is to link specific genetic variants with specific phenotypes. This information can then be used to evaluate the risk or susceptibility of a population to develop a certain disease, in diagnostic tests or to guide treatments. Indeed, single nucleotide polymorphisms
(SNPs) within non-coding region of GPM6A have been linked to schizophrenia (Boks et al., 2008; Schizophrenia Working Group of the Psychiatric Genomics Consortium, 2014; Li et al., 2017; Pardiñas et al., 2018; Lam et al., 2019), and one variant for both neuroticism (Nagel et al., 2018) and bipolar disorder (Greenwood et al., 2012). However, there are still no reports confirming whether any of these SNPs lead to changes in M6a gene expression or function. Our lab studied 3 non-synonymous SNPs within GPM6A's TMD coding region reported in the dbSNP database ${ }^{3}$. By doing reverse genetic experiments, we demonstrated that all nsSNPs prevented M6a from being functional in neurons, impaired formation of dendritic spines and synapses owing to decreased stability, dimerization, or improper folding of the protein (Formoso et al., 2015b, 2016).

\section{CONCLUDING REMARKS}

Evidence collected over 30 years since its discovery show that glycoprotein M6a has a critical role in synapse formation,

\footnotetext{
${ }^{3}$ https://www.ncbi.nlm.nih.gov/snp/
} 
plasticity, and maintenance. Previous research so far has focused on in vitro approaches, with only a few articles studying M6a-deficient mice. Unfortunately, in none of those studies the synaptic activity or synaptic integrity were interrogated. New data coming from "omic" and GWAS approaches in combination with basic investigation will expand our knowledge of the field and define the exact role of GPM6A in neuronal development and synaptopathies. This in turn will offer new routes to improve diagnosis and develop more effective treatments.

\section{AUTHOR CONTRIBUTIONS}

$\mathrm{AL}, \mathrm{GA}$, and CS were involved in bibliography revision. CS contributed to the design and conceptualization of the research topic. CS, AL, and GA wrote the manuscript. AL and GA made the figure and the table. All authors contributed to the article and approved the submitted version.

\section{REFERENCES}

Abraham, J. R., Szoko, N., Barnard, J., Rubin, R. A., Schlatzer, D., Lundberg, K., et al. (2019). Proteomic Investigations of Autism Brain Identify Known and Novel Pathogenetic Processes. Sci. Rep. 9:13118.

Alfonso, J., Aguero, F., Sanchez, D. O., Flugge, G., Fuchs, E., Frasch, A. C., et al. (2004a). Gene expression analysis in the hippocampal formation of tree shrews chronically treated with cortisol. J. Neurosci. Res. 78, 702-710. doi: 10.1002/jnr. 20328

Alfonso, J., Pollevick, G. D., Van Der Hart, M. G., Flugge, G., Fuchs, E., and Frasch, A. C. (2004b). Identification of genes regulated by chronic psychosocial stress and antidepressant treatment in the hippocampus. Eur. J. Neurosci. 19, 659-666. doi: 10.1111/j.1460-9568.2004.03178.x

Alfonso, J., Fernandez, M. E., Cooper, B., Flugge, G., and Frasch, A. C. (2005). The stress-regulated protein M6a is a key modulator for neurite outgrowth and filopodium/spine formation. Proc. Natl. Acad. Sci. U. S. A. 102, 17196-17201. doi: 10.1073/pnas.0504262102

Alfonso, J., Frick, L. R., Silberman, D. M., Palumbo, M. L., Genaro, A. M., and Frasch, A. C. (2006). Regulation of hippocampal gene expression is conserved in two species subjected to different stressors and antidepressant treatments. Biol. Psychiatry 59, 244-251. doi: 10.1016/j.biopsych.2005. 06.036

Alfonso, J., Pollevick, G. D., Castensson, A., Jazin, E., and Frasch, A. C. (2002). Analysis of gene expression in the rat hippocampus using Real Time PCR reveals high inter-individual variation in mRNA expression levels. J. Neurosci. Res. 67, 225-234. doi: 10.1002/jnr.10105

Alvarez Julia, A., Frasch, A. C., and Fuchsova, B. (2016). Neuronal filopodium formation induced by the membrane glycoprotein M6a (Gpm6a) is facilitated by coronin-1a, Rac1, and p21-activated kinase 1 (Pak1). J. Neurochem. 137, 46-61. doi: 10.1111/jnc. 13552

Andrews, M. R., Czvitkovich, S., Dassie, E., Vogelaar, C. F., Faissner, A., Blits, B., et al. (2009). Alpha9 integrin promotes neurite outgrowth on tenascinC and enhances sensory axon regeneration. J. Neurosci. 29, 5546-5557. doi: 10.1523/jneurosci.0759-09.2009

Antonucci, F., Corradini, I., Fossati, G., Tomasoni, R., Menna, E., and Matteoli, M. (2016). SNAP-25, a Known Presynaptic Protein with Emerging Postsynaptic Functions. Front. Synaptic Neurosci. 8:7. doi: 10.3389/fnsyn.2016.00007

Aparicio, G. I., Formoso, K., Leon, A., Frasch, A. C., and Scorticati, C. (2020). Identification of Potential Interacting Proteins With the Extracellular Loops of the Neuronal Glycoprotein M6a by TMT/MS. Front. Synaptic Neurosci. 12:28. doi: $10.3389 /$ fnsyn.2020.00028

Baumrind, N. L., Parkinson, D., Wayne, D. B., Heuser, J. E., and Pearlman, A. L. (1992). EMA: a developmentally regulated cell-surface glycoprotein of CNS

\section{FUNDING}

AL and GA were Ph.D. students. CS was a researcher from the National Council for Research of Argentina (CONICET). This work was supported by Agencia Nacional de Promoción Científica y Tecnológica (ANPCyT) BID-ANPCyT-PICT 20161223 and BID-ANCyT-PICT 2019-1051 to CS and BIDANPCyT-PICT 2017-1736.

\section{ACKNOWLEDGMENTS}

We gratefully thank Dr. Karina Formoso (Institute of Metabolic and Cardiovascular Diseases, Université de Toulouse III, ISERM), Dr. Mariano M. Boccia (Laboratorio de Neurofarmacología de los Procesos de Memoria, Facultad de Farmacia y Bioquímica, UBA) and Dr. Fernán Agüero (Bioinformatics Group leader at IIBio-CONICET-UNSAM) who critically read the manuscript.

neurons that is concentrated at the leading edge of growth cones. Dev. Dyn. 194, 311-325. doi: 10.1002/aja.1001940407

Beesley, P. W., Herrera-Molina, R., Smalla, K. H., and Seidenbecher, C. (2014). The Neuroplastin adhesion molecules: key regulators of neuronal plasticity and synaptic function. J. Neurochem. 131, 268-283. doi: 10.1111/jnc.12816

Bian, W. J., Miao, W. Y., He, S. J., Qiu, Z., and Yu, X. (2015). Coordinated Spine Pruning and Maturation Mediated by Inter-Spine Competition for Cadherin/Catenin Complexes. Cell 162, 808-822. doi: 10.1016/j.cell.2015.07. 018

Boks, M. P., Hoogendoorn, M., Jungerius, B. J., Bakker, S. C., Sommer, I. E., Sinke, R. J., et al. (2008). Do mood symptoms subdivide the schizophrenia phenotype? Association of the GMP6A gene with a depression subgroup. Am. J. Med. Genet. B Neuropsychiatr. Genet. 147B, 707-711. doi: 10.1002/ajmg.b.30667

Boyken, J., Gronborg, M., Riedel, D., Urlaub, H., Jahn, R., and Chua, J. J. (2013). Molecular profiling of synaptic vesicle docking sites reveals novel proteins but few differences between glutamatergic and GABAergic synapses. Neuron 78, 285-297. doi: 10.1016/j.neuron.2013.02.027

Brocco, M. A., Fernandez, M. E., and Frasch, A. C. (2010). Filopodial protrusions induced by glycoprotein M6a exhibit high motility and aids synapse formation. Eur. J. Neurosci. 31, 195-202. doi: 10.1111/j.1460-9568.2009.07064.x

Caceres, A., Ye, B., and Dotti, C. G. (2012). Neuronal polarity: demarcation, growth and commitment. Curr. Opin. Cell Biol. 24, 547-553. doi: 10.1016/j.ceb.2012. 05.011

Chiasserini, D., Bijnsdorp, I., Bellomo, G., Orvietani, P. L., Piersma, S. R., Pham, T., et al. (2020). Proteomic analysis of extracellular vesicles in cerebrospinal fluid of patients with Alzheimer's disease. medRxiv. [Preprint] doi: 10.1101/2020.02. 22.20026609

Claussnitzer, M., Cho, J. H., Collins, R., Cox, N. J., Dermitzakis, E. T., Hurles, M. E., et al. (2020). A brief history of human disease genetics. Nature 577, 179-189.

Cooper, B., Werner, H. B., and Flugge, G. (2008). Glycoprotein M6a is present in glutamatergic axons in adult rat forebrain and cerebellum. Brain Res. 1197, 1-12. doi: 10.1016/j.brainres.2007.11.066

Egbujo, C. N., Sinclair, D., and Hahn, C. G. (2016). Dysregulations of Synaptic Vesicle Trafficking in Schizophrenia. Curr. Psychiatry Rep. 18, 77.

El-Kordi, A., Kastner, A., Grube, S., Klugmann, M., Begemann, M., Sperling, S., et al. (2013). A single gene defect causing claustrophobia. Transl. Psychiatry 3, e254. doi: 10.1038/tp.2013.28

Fagerberg, L., Hallstrom, B. M., Oksvold, P., Kampf, C., Djureinovic, D., Odeberg, J., et al. (2014). Analysis of the human tissue-specific expression by genomewide integration of transcriptomics and antibody-based proteomics. Mol. Cell Proteomics 13, 397-406. doi: 10.1074/mcp.m113.035600

Fang, P., Wang, X. J., Xue, Y., Liu, M. Q., Zeng, W. F., Zhang, Y., et al. (2016). In-depth mapping of the mouse brain $\mathrm{N}$-glycoproteome reveals widespread 
N-glycosylation of diverse brain proteins. Oncotarget 7, 38796-38809. doi: 10.18632/oncotarget.9737

Fernandez, M. E., Alfonso, J., Brocco, M. A., and Frasch, A. C. (2010). Conserved cellular function and stress-mediated regulation among members of the proteolipid protein family. J. Neurosci. Res. 88, 1298-1308.

Formoso, K., Billi, S. C., Frasch, A. C., and Scorticati, C. (2015a). Tyrosine 251 at the C-terminus of neuronal glycoprotein M6a is critical for neurite outgrowth. J. Neurosci. Res. 93, 215-229. doi: 10.1002/jnr.23482

Formoso, K., Garcia, M. D., Frasch, A. C., and Scorticati, C. (2015b). Filopodia formation driven by membrane glycoprotein M6a depends on the interaction of its transmembrane domains. J. Neurochem. 134, 499-512. doi: 10.1111/jnc. 13153

Formoso, K., Garcia, M. D., Frasch, A. C., and Scorticati, C. (2016). Evidence for a role of glycoprotein M6a in dendritic spine formation and synaptogenesis. Mol. Cell Neurosci. 77, 95-104. doi: 10.1016/j.mcn.2016.10.005

Fuchsova, B., Alvarez Julia, A., Rizavi, H. S., Frasch, A. C., and Pandey, G. N. (2015). Altered expression of neuroplasticity-related genes in the brain of depressed suicides. Neuroscience 299, 1-17. doi: 10.1016/j.neuroscience.2015.04.057

Fuchsova, B., Fernandez, M. E., Alfonso, J., and Frasch, A. C. (2009). Cysteine residues in the large extracellular loop (EC2) are essential for the function of the stress-regulated glycoprotein M6a. J. Biol. Chem. 284, 32075-32088. doi: 10.1074/jbc.m109.012377

Fuentes, F., and Arregui, C. O. (2009). Microtubule and cell contact dependency of ER-bound PTP1B localization in growth cones. Mol. Biol. Cell 20, 1878-1889. doi: 10.1091/mbc.e08-07-0675

Garcia, M. D., Formoso, K., Aparicio, G. I., Frasch, A. C. C., and Scorticati, C. (2017). The Membrane Glycoprotein M6a Endocytic/Recycling Pathway Involves Clathrin-Mediated Endocytosis and Affects Neuronal Synapses. Front. Mol. Neurosci. 10:296. doi: 10.3389/fnmol.2017. 00296

Greenwood, T. A., Akiskal, H. S., Akiskal, K. K., Bipolar Genome, S., and Kelsoe, J. R. (2012). Genome-wide association study of temperament in bipolar disorder reveals significant associations with three novel Loci. Biol. Psychiatry 72, 303310. doi: 10.1016/j.biopsych.2012.01.018

Greer, J. M., and Lees, M. B. (2002). Myelin proteolipid protein-the first 50 years. Int. J. Biochem. Cell Biol. 34, 211-215. doi: 10.1016/s1357-2725(01) 00136-4

Gregor, A., Kramer, J. M., Van Der Voet, M., Schanze, I., Uebe, S., Donders, R., et al. (2014). Altered GPM6A/M6 dosage impairs cognition and causes phenotypes responsive to cholesterol in human and Drosophila. Hum. Mutat. 35, 1495-1505. doi: 10.1002/humu.22697

Grunewald, L., Chiocchetti, A. G., Weber, H., Scholz, C. J., Schartner, C., Freudenberg, F., et al. (2021). Knockdown of the ADHD Candidate Gene Diras2 in Murine Hippocampal Primary Cells. J. Atten. Disord. 25, 572-583. doi: $10.1177 / 1087054718822129$

Haenig, C., Atias, N., Taylor, A. K., Mazza, A., Schaefer, M. H., Russ, J., et al. (2020). Interactome Mapping Provides a Network of Neurodegenerative Disease Proteins and Uncovers Widespread Protein Aggregation in Affected Brains. Cell Rep. 32:108050. doi: 10.1016/j.celrep.2020.108050

Hida, Y., and Ohtsuka, T. (2010). CAST and ELKS proteins: structural and functional determinants of the presynaptic active zone. J. Biochem. 148, 131137. doi: $10.1093 / \mathrm{jb} / \mathrm{mvq} 065$

Honda, A., Ito, Y., Takahashi-Niki, K., Matsushita, N., Nozumi, M., Tabata, H., et al. (2017). Extracellular Signals Induce Glycoprotein M6a Clustering of Lipid Rafts and Associated Signaling Molecules. J. Neurosci. 37, 4046-4064. doi: 10.1523/jneurosci.3319-16.2017

Huminiecki, L., Lloyd, A. T., and Wolfe, K. H. (2003). Congruence of tissue expression profiles from Gene Expression Atlas, SAGEmap and TissueInfo databases. BMC Genomics 4:31. doi: 10.1186/1471-2164-4-31

Huttlin, E. L., Bruckner, R. J., Paulo, J. A., Cannon, J. R., Ting, L., Baltier, K., et al. (2017). Architecture of the human interactome defines protein communities and disease networks. Nature 545, 505-509. doi: 10.1038/nature22366

Ito, Y., Honda, A., and Igarashi, M. (2018). Glycoprotein M6a as a signaling transducer in neuronal lipid rafts. Neurosci. Res. 128, 19-24. doi: 10.1016/j. neures.2017.11.002

Jahn, O., Siems, S. B., Kusch, K., Hesse, D., Jung, R. B., Liepold, T., et al. (2020). The CNS Myelin Proteome: deep Profile and Persistence After Post-mortem Delay. Front. Cell Neurosci. 14:239. doi: 10.3389/fncel.2020.00239
Kang, R., Wan, J., Arstikaitis, P., Takahashi, H., Huang, K., Bailey, A. O., et al. (2008). Neural palmitoyl-proteomics reveals dynamic synaptic palmitoylation. Nature 456, 904-909. doi: 10.1038/nature07605

Lachén-Montes, M., Gonzalez-Morales, A., De Morentin, X. M., PerezValderrama, E., Ausin, K., Zelaya, M. V., et al. (2016). An early dysregulation of FAK and MEK/ERK signaling pathways precedes the beta-amyloid deposition in the olfactory bulb of APP/PS1 mouse model of Alzheimer's disease. J. Proteomics 148, 149-158. doi: 10.1016/j.jprot.2016.07.032

Lagenaur, C., Kunemund, V., Fischer, G., Fushiki, S., and Schachner, M. (1992). Monoclonal M6 antibody interferes with neurite extension of cultured neurons. J. Neurobiol. 23, 71-88. doi: 10.1002/neu.480230108

Lam, M., Chen, C. Y., Li, Z., Martin, A. R., Bryois, J., Ma, X., et al. (2019). Comparative genetic architectures of schizophrenia in East Asian and European populations. Nat. Genet. 51, 1670-1678.

Li, Z., Chen, J., Yu, H., He, L., Xu, Y., Zhang, D., et al. (2017). Genome-wide association analysis identifies 30 new susceptibility loci for schizophrenia. Nat. Genet. 49, 1576-1583.

Liu, Y. T., Tao, C. L., Lau, P. M., Zhou, Z. H., and Bi, G. Q. (2019). Postsynaptic protein organization revealed by electron microscopy. Curr. Opin. Struct. Biol. 54, 152-160. doi: 10.1016/j.sbi.2019.02.012

Martorella, M., Barford, K., Winkler, B., and Deppmann, C. D. (2017). Emergent Role of Coronin-1a in Neuronal Signaling. Vitam. Horm. 104, 113-131. doi: 10.1016/bs.vh.2016.10.002

Michibata, H., Okuno, T., Konishi, N., Kyono, K., Wakimoto, K., Aoki, K., et al. (2009). Human GPM6A is associated with differentiation and neuronal migration of neurons derived from human embryonic stem cells. Stem Cells Dev. 18, 629-639. doi: 10.1089/scd.2008.0215

Miller, M., and Peters, A. (1981). Maturation of rat visual cortex. II. A combined Golgi-electron microscope study of pyramidal neurons. J. Comp. Neurol. 203, 555-573. doi: 10.1002/cne. 902030402

Mita, S., De Monasterio-Schrader, P., Funfschilling, U., Kawasaki, T., Mizuno, H., Iwasato, T., et al. (2015). Transcallosal Projections Require Glycoprotein M6-Dependent Neurite Growth and Guidance. Cereb. Cortex 25, 4111-4125. doi: 10.1093/cercor/bhu129

Miura, R., Ethell, I. M., and Yamaguchi, Y. (2001). Carbohydrate-protein interactions between HNK-1-reactive sulfoglucuronyl glycolipids and the proteoglycan lectin domain mediate neuronal cell adhesion and neurite outgrowth. J. Neurochem. 76, 413-424. doi: 10.1046/j.1471-4159.2001.00042.x

Monteleone, M. C., Adrover, E., Pallares, M. E., Antonelli, M. C., Frasch, A. C., and Brocco, M. A. (2014). Prenatal stress changes the glycoprotein GPM6A gene expression and induces epigenetic changes in rat offspring brain. Epigenetics 9 , 152-160. doi: 10.4161/epi.25925

Monteleone, M. C., Billi, S. C., Viale, L., Catoira, N. P., Frasch, A. C., and Brocco, M. A. (2020). Search of brain-enriched proteins in salivary extracellular vesicles for their use as mental disease biomarkers: a pilot study of the neuronal glycoprotein M6a. J. Affect. Disord. Rep. 1:100003 doi: 10.1016/j.jadr.2020. 100003

Mukobata, S., Hibino, T., Sugiyama, A., Urano, Y., Inatomi, A., Kanai, Y., et al. (2002). M6a acts as a nerve growth factor-gated $\mathrm{Ca}(2+)$ channel in neuronal differentiation. Biochem. Biophys. Res. Commun. 297, 722-728. doi: 10.1016/ s0006-291x(02)02284-2

Muraoka, S., Deleo, A. M., Sethi, M. K., Yukawa-Takamatsu, K., Yang, Z., Ko, J., et al. (2020). Proteomic and biological profiling of extracellular vesicles from Alzheimer's disease human brain tissues. Alzheimers Dement. 16, 896-907. doi: 10.1002/alz.12089

Nagel, M., Jansen, P. R., Stringer, S., Watanabe, K., De Leeuw, C. A., Bryois, J., et al. (2018). Meta-analysis of genome-wide association studies for neuroticism in 449,484 individuals identifies novel genetic loci and pathways. Nat. Genet. 50, 920-927.

Neugebauer, K. M., Tomaselli, K. J., Lilien, J., and Reichardt, L. F. (1988). $\mathrm{N}$-cadherin, NCAM, and integrins promote retinal neurite outgrowth on astrocytes in vitro. J. Cell Biol. 107, 1177-1187. doi: 10.1083/jcb.107.3.1177

Nosov, G., Kahms, M., and Klingauf, J. (2020). The Decade of Super-Resolution Microscopy of the Presynapse. Front. Synaptic Neurosci. 12:32. doi: 10.3389/ fnsyn.2020.00032

Olinsky, S., Loop, B. T., Dekosky, A., Ripepi, B., Weng, W., Cummins, J., et al. (1996). Chromosomal mapping of the human M6 genes. Genomics 33, 532-536. doi: 10.1006/geno.1996.0231 
Ozcan, A. S. (2017). Filopodia: a Rapid Structural Plasticity Substrate for Fast Learning. Front. Synaptic Neurosci. 9:12. doi: 10.3389/fnsyn.2017.00012

Pardiñas, A. F., Holmans, P., Pocklington, A. J., Escott-Price, V., Ripke, S., Carrera, N., et al. (2018). Common schizophrenia alleles are enriched in mutationintolerant genes and in regions under strong background selection. Nat. Genet. 50, 381-389.

Penzes, P., Cahill, M. E., Jones, K. A., Vanleeuwen, J. E., and Woolfrey, K. M. (2011). Dendritic spine pathology in neuropsychiatric disorders. Nat. Neurosci. 14, 285-293. doi: 10.1038/nn.2741

Pourhaghighi, R., Ash, P. E. A., Phanse, S., Goebels, F., Hu, L. Z. M., Chen, S., et al. (2020). BraInMap Elucidates the Macromolecular Connectivity Landscape of Mammalian Brain. Cell Syst. 10:e314.

Quiroz-Baez, R., Hernandez-Ortega, K., and Martinez-Martinez, E. (2020). Insights into the proteomic profiling of extracellular vesicles for the identification of early biomarkers of neurodegeneration. Front. Neurol. 11:580030. doi: 10.3389/fneur.2020.580030

Rao-Ruiz, P., Carney, K. E., Pandya, N., Van Der Loo, R. J., Verheijen, M. H., Van Nierop, P., et al. (2015). Time-dependent changes in the mouse hippocampal synaptic membrane proteome after contextual fear conditioning. Hippocampus 25, 1250-1261. doi: 10.1002/hipo.22432

Reim, D., Distler, U., Halbedl, S., Verpelli, C., Sala, C., Bockmann, J., et al. (2017). Proteomic Analysis of Post-synaptic Density Fractions from Shank3 Mutant Mice Reveals Brain Region Specific Changes Relevant to Autism Spectrum Disorder. Front. Mol. Neurosci. 10:26. doi: 10.3389/fnmol.2017. 00026

Rosas, N. M., Alvarez Julia, A., Alzuri, S. E., Frasch, A. C., and Fuchsova, B. (2018). Alanine Scanning Mutagenesis of the C-Terminal Cytosolic End of Gpm6a Identifies Key Residues Essential for the Formation of Filopodia. Front. Mol. Neurosci. 11:314. doi: 10.3389/fnmol.2018.00314

Roussel, G., Trifilieff, E., Lagenaur, C., and Nussbaum, J. L. (1998). Immunoelectron microscopic localization of the M6a antigen in rat brain. J. Neurocytol. 27, 695-703.

Sato, Y., Mita, S., Fukushima, N., Fujisawa, H., Saga, Y., and Hirata, T. (2011a). Induction of axon growth arrest without growth cone collapse through the $\mathrm{N}$-terminal region of four-transmembrane glycoprotein M6a. Dev. Neurobiol. 71, 733-746. doi: 10.1002/dneu.20941

Sato, Y., Watanabe, N., Fukushima, N., Mita, S., and Hirata, T. (2011b). Actinindependent behavior and membrane deformation exhibited by the fourtransmembrane protein M6a. PLoS One 6:e26702. doi: 10.1371/journal.pone. 0026702

Schizophrenia Working Group of the Psychiatric Genomics Consortium (2014). Biological insights from 108 schizophrenia-associated genetic loci. Nature 511, 421-427. doi: 10.1038/nature13595

Scorticati, C., Formoso, K., and Frasch, A. C. (2011). Neuronal glycoprotein M6a induces filopodia formation via association with cholesterol-rich lipid rafts. J. Neurochem. 119, 521-531. doi: 10.1111/j.1471-4159.2011.07252.x

Sengpiel, F. (2018). Overview: neuroplasticity and synaptic function in neuropsychiatric disorders. J. Physiol. 596, 2745-2746. doi: 10.1113/jp275940

Shen, K., and Cowan, C. W. (2010). Guidance molecules in synapse formation and plasticity. Cold Spring Harb. Perspect. Biol. 2:a001842. doi: 10.1101/cshperspect. a001842

Sotelo, C., Hillman, D. E., Zamora, A. J., and Llinas, R. (1975). Climbing fiber deafferentation: its action on Purkinje cell dendritic spines. Brain Res. 98, 574-581. doi: 10.1016/0006-8993(75)90374-1

Takamori, S., Holt, M., Stenius, K., Lemke, E. A., Gronborg, M., Riedel, D., et al. (2006). Molecular anatomy of a trafficking organelle. Cell 127, 831-846.
Taoufiq, Z., Ninov, M., Villar-Briones, A., Wang, H. Y., Sasaki, T., Roy, M. C., et al. (2020). Hidden proteome of synaptic vesicles in the mammalian brain. Proc. Natl. Acad. Sci. U. S. A. 117, 33586-33596. doi: 10.1073/pnas.2011870117

Tonnesen, J., and Nagerl, U. V. (2016). Dendritic Spines as Tunable Regulators of Synaptic Signals. Front. Psychiatry 7:101. doi: 10.3389/fpsyt.2016.00101

Toro, R., Konyukh, M., Delorme, R., Leblond, C., Chaste, P., Fauchereau, F., et al. (2010). Key role for gene dosage and synaptic homeostasis in autism spectrum disorders. Trends Genet. 26, 363-372. doi: 10.1016/j.tig.2010.05.007

Torres, V. I., Vallejo, D., and Inestrosa, N. C. (2017). Emerging Synaptic Molecules as Candidates in the Etiology of Neurological Disorders. Neural Plast. 2017:8081758.

Tu, Y. K., Duman, J. G., and Tolias, K. F. (2018). The Adhesion-GPCR BAI1 Promotes Excitatory Synaptogenesis by Coordinating Bidirectional Transsynaptic Signaling. J. Neurosci. 38, 8388-8406. doi: 10.1523/jneurosci.3461-17. 2018

Ulloa, F., Cotrufo, T., Ricolo, D., Soriano, E., and Araujo, S. J. (2018). SNARE complex in axonal guidance and neuroregeneration. Neural Regen. Res. 13, 386-392. doi: 10.4103/1673-5374.228710

Werner, H. B., Kramer-Albers, E. M., Strenzke, N., Saher, G., Tenzer, S., OhnoIwashita, Y., et al. (2013). A critical role for the cholesterol-associated proteolipids PLP and M6B in myelination of the central nervous system. Glia 61, 567-586. doi: 10.1002/glia.22456

Wu, D. F., Koch, T., Liang, Y. J., Stumm, R., Schulz, S., Schroder, H., et al. (2007). Membrane glycoprotein M6a interacts with the micro-opioid receptor and facilitates receptor endocytosis and recycling. J. Biol. Chem. 282, 22239-22247. doi: $10.1074 /$ jbc.m700941200

Xu, P. T., Li, Y. J., Qin, X. J., Scherzer, C. R., Xu, H., Schmechel, D. E., et al. (2006). Differences in apolipoprotein E3/3 and E4/4 allele-specific gene expression in hippocampus in Alzheimer disease. Neurobiol. Dis. 21, 256-275. doi: 10.1016/ j.nbd.2005.07.004

Yu, Y., Fuscoe, J. C., Zhao, C., Guo, C., Jia, M., Qing, T., et al. (2014). A rat RNASeq transcriptomic BodyMap across 11 organs and 4 developmental stages. Nat. Commun. 5:3230.

Yue, F., Cheng, Y., Breschi, A., Vierstra, J., Wu, W., Ryba, T., et al. (2014). A comparative encyclopedia of DNA elements in the mouse genome. Nature 515, 355-364.

Yuste, R., and Bonhoeffer, T. (2004). Genesis of dendritic spines: insights from ultrastructural and imaging studies. Nat. Rev. Neurosci. 5, 24-34. doi: 10.1038/ nrn 1300

Zhao, J., Iida, A., Ouchi, Y., Satoh, S., and Watanabe, S. (2008). M6a is expressed in the murine neural retina and regulates neurite extension. Mol. Vis. 14, $1623-1630$.

Ziv, N. E., and Fisher-Lavie, A. (2014). Presynaptic and postsynaptic scaffolds: dynamics fast and slow. Neuroscientist 20, 439-452. doi: $10.1177 / 1073858414523321$

Conflict of Interest: The authors declare that the research was conducted in the absence of any commercial or financial relationships that could be construed as a potential conflict of interest.

Copyright (c) 2021 León, Aparicio and Scorticati. This is an open-access article distributed under the terms of the Creative Commons Attribution License (CC BY). The use, distribution or reproduction in other forums is permitted, provided the original author(s) and the copyright owner(s) are credited and that the original publication in this journal is cited, in accordance with accepted academic practice. No use, distribution or reproduction is permitted which does not comply with these terms. 